\title{
Common mode rejection in electrically coupled MEMS resonators utilizing mode localization for sensor applications
}

\author{
P. Thiruvenkatanathan, J. Yan, and A. A. Seshia* \\ Department of Engineering, \\ University of Cambridge, \\ Cambridge, UK. \\ *email: aas41@cam.ac.uk
}

\begin{abstract}
Measuring shifts in eigenstates due to vibration localization in an array of weakly coupled resonators offer two distinct advantages for sensor applications as opposed to the technique of simply measuring resonant frequency shifts: (1) orders of magnitude enhancement in parametric sensitivity and (2) intrinsic common mode rejection. In this paper, we experimentally demonstrate the common mode rejection capabilities of such sensors. The vibration behavior is studied in pairs of nearly identical MEMS resonators that are electrically coupled, and subjected to small perturbations in stiffness under different ambient pressure and temperature. The shifts in the eigenstates for the same parametric perturbation in stiffness are experimentally demonstrated to be over three orders of magnitude greater than corresponding resonant frequency variations. They are also shown to remain relatively constant to variations in ambient temperature and pressure. This increased relative robustness to environmental drift, along with the advantage of ultra-high parametric sensitivity, opens the door to an alternative approach to achieving higher sensitivity and stability in micromechanical sensors.
\end{abstract}

\section{INTRODUCTION}

The perennial challenge of good sensor design has been maximizing the sensitivity of the sensor to the measurand while minimizing its sensitivity to other variables. While resonant frequency based sensors have emerged as promising alternatives for the detection of small, linear structural perturbations in micromechanical structures, an uncompensated resonant frequency output makes such sensors sensitive not only to the factor to be measured, but also to other unwanted environmental drift (e.g.: fluctuations in ambient temperature and pressure). Elaborate temperature control or compensation techniques are usually required to attain sufficient accuracy in such sensors. Furthermore, the resolution of resonant frequency based sensors depends on the quality factor $(\mathrm{Q})$ of resonance thereby often constraining the design of such sensors to topologies that yield high Q [1].

Various techniques of compensating for environmental drift on the resonant frequency (specifically due to temperature and pressure) have been proposed. Most temperature compensation techniques rely on an active compensation approach wherein a temperature sensor is placed in the vicinity of the MEMS resonator to correct for temperature drift in the output frequency [2]. Alternate compensation methods involve designing the resonators to be insensitive to temperature. This may be achieved by building mechanisms with temperature sensitive materials of different thermal expansion coefficients that apply a corrective force to compensate for the temperature induced stresses and consequently their frequency dependences on the resonator [3]. Alternative approaches like the dual-mode compensation technique similar to the one used in quartz resonators [4] and using the quality factor as a thermometer in order to compensate for temperature drifts [5] have also been reported.

In what follows, we propose the use of an alternate technique of sensing to attain outputs that are not only insensitive to unwanted environmental drifts such as temperature and pressure, but are also orders of magnitude more sensitive to the desired load compared to corresponding resonant frequency variations. Furthermore, the outputs are also shown to be relatively independent of Q thereby providing an added advantage in terms of the design of micromechanical sensors.

When mechanically identical resonators are coupled through weak springs, the vibrations propagate evenly throughout the structure due to the periodicity of the system. Any structural perturbation in one of the resonators however, breaks the periodicity of the system thereby leading to (sometimes severe) confinement of vibration energy to certain geometric regions of the system. The extent of such vibration energy confinement or localization depends not only on the magnitude of the periodicity breaking irregularity, but also on the strength of the internal coupling spring constant between the resonators with weaker coupling leading to stronger localization. Measuring the shifts in the eigenstates due to such vibration localization can consequently, yield sensitivities as high as orders of magnitude greater than corresponding resonant frequency variations under conditions of weak internal coupling [6]-[8]. Furthermore, since each of the coupled resonators in the array are subjected to the same ambient environmental conditions (common mode) but only one of the coupled structures is subjected to an induced load (differential mode), we get an output that is highly sensitive to the measurand but relatively insensitive to other variables.

This research is funded in part by the British Council (UKIERI), Churchill College and the Cambridge Commonwealth Trust (University of Cambridge, UK.) 


\section{THEORY}

Consider two resonators coupled through a weak coupling spring $\left(k_{c}\right)$ as represented in the discretized model shown in fig. 1 .

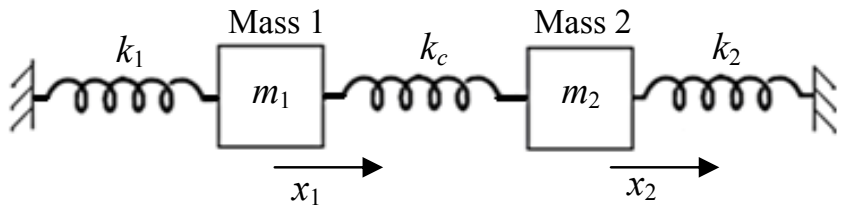

Figure 1: Lumped element model of a coupled two degree-of-freedom spring mass system.

When the two resonators are perfectly identical $\left(m_{1}=m_{2}=m\right.$; $k_{1}=k_{2}=k$ ) the system is symmetric about the coupling spring and the vibrations propagate evenly throughout the system. Any structural perturbation on one of the resonators however, disturbs the symmetry of the system thereby inducing a localization of the vibration modes. The variation in the eigenstates owing to an induced perturbation in the stiffness of one of the resonators may be expressed as [8]

$$
\left|\frac{u_{n}-u_{0 n}}{u_{0 n}}\right| \cong\left|\frac{\Delta k}{4 k_{c}}\right| ;(n=1,2) \text {. }
$$

where $u_{\mathrm{n}}$ and $u_{0 \mathrm{n}}$ represent the perturbed and unperturbed non-dimensionalized two-component eigenvectors (eigenstates) that are deduced from the amplitudes of vibration of each of the coupled resonators at the two fundamental modes. $\Delta k$ represents the induced stiffness perturbation in one of the coupled resonators and $n$ denotes the fundamental mode of vibration. Comparing this with the relative shift in the resonant frequency for the same induced perturbation in stiffness -

$$
\left|\frac{f-f_{0}}{f_{0}}\right| \cong\left|\frac{\Delta k}{2 k}\right|,
$$

it can be noticed that for any value of $k_{c}<k / 2$, the relative shifts in the eigenstates are more sensitive than those in frequency. In equation (2), $f_{0}$ and $f$ represent the frequency before and after inducing a stiffness perturbation.

It can be observed from equation (1) that reducing the strength of internal coupling between the resonators leads to an enhancement of the parametric sensitivity. Sensitivity enhancements exceeding two orders of magnitude have been experimentally demonstrated by employing an electrical coupling technique [8]. Electrical coupling is a passive coupling technique that has been used to attain weak coupling springs for MEMS filters [9]. When two resonators are separated by a small coupling gap and subjected to different DC polarization voltages, the displacement-dependent component of the attractive electrostatic force generated between the resonators results in a mechanical spring-like behaviour. The direction of this displacement-dependent component is opposite to the force and hence leads to the formation of a negative electrostatic coupling spring that is voltage tunable. We use this technique of coupling, to attain very low magnitudes of $k_{c}$ and in consequence high parametric sensitivities. Sensitivity enhancements exceeding three orders compared to corresponding resonant frequency variations are demonstrated here for the first time.

From equation (1), it can also be observed that since the eigenstates are deduced from the amplitudes of vibration of both the coupled resonators at the eigenvalues, any effects on the stiffness due to ambient environmental fluctuations (e.g.: temperature) would affect both the identical resonators to the same extent thereby leading to a common mode cancellation of these effects to the first order. However, any changes in the stiffness on one of the resonators relative to the other (differential mode), leads to significant shifts in the eigenstates under conditions of weak internal coupling as expressed in equation (1). Such a common mode rejection capability enables the realization of sensors that are highly sensitive to the measurand alone without employing any active/passive control or compensation techniques, making this form a sensing particularly attractive over the more conventional resonant frequency based sensing approach. This phenomenon is demonstrated here by subjecting pairs of electrically coupled MEMS resonators to small periodicity breaking stiffness perturbations in one of the coupled structures and comparing the corresponding relative shifts observed in the eigenstates and resonant frequency under varying ambient temperature and pressure.

\section{EXPERIMENT}

\section{A. Device descriptions and characterization}

Two different resonator configurations are used to demonstrate the sensitivity enhancements and common mode rejection capabilities of such sensors - a pair of electrically coupled nearly identical double ended tuning forks (DETFs) and a pair of electrically coupled nearly identical flexural wine glass mode ring resonators. Optical micrographs of the ring and DETF configurations are shown in figs. 2 and 3 respectively. Both devices were fabricated in a commercial foundry process using the silicon-on-insulator microelectromechanical systems (SOI-MEMS) process through MEMSCAP Inc., USA.

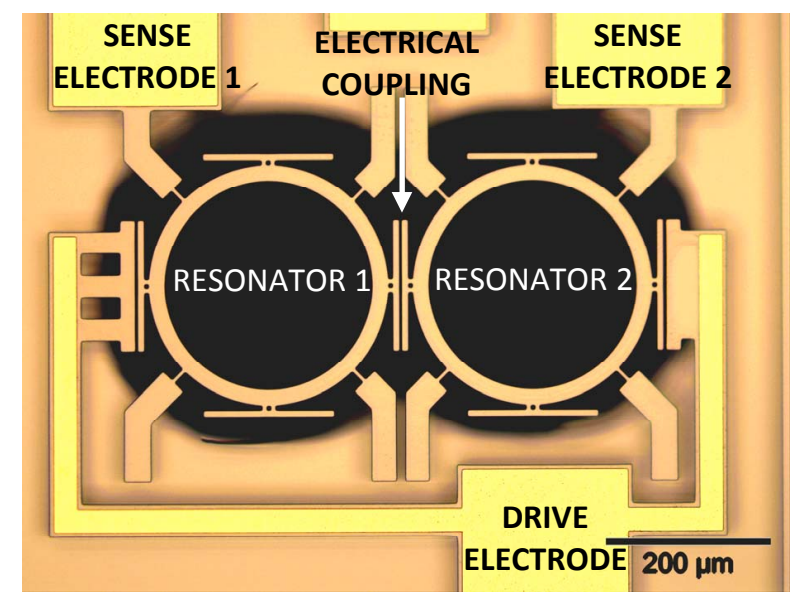

Figure 2: Optical micrograph of a pair of electrically coupled flexural wine glass mode ring resonators. 


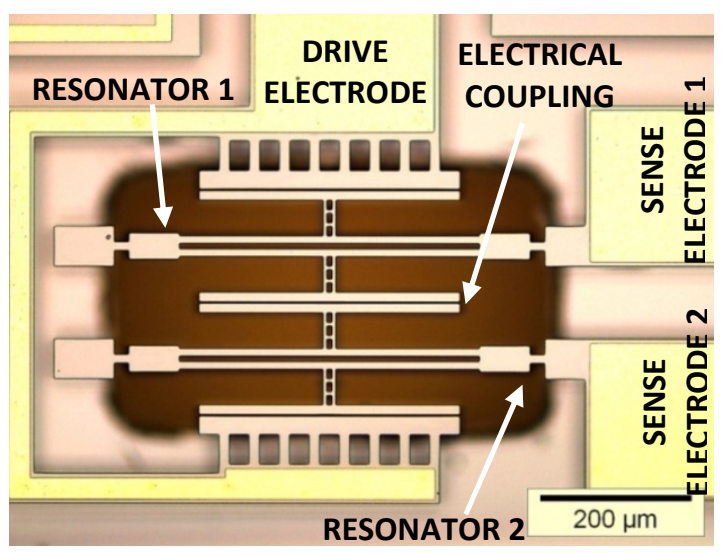

Figure 3: Optical micrograph of a pair of electrically coupled double ended tuning fork (DETF) resonators.

Each of the tines in the DETF configuration was designed to be $25 \mu \mathrm{m}$ thick, $300 \mu \mathrm{m}$ long and $6 \mu \mathrm{m}$ wide with a gap of $6 \mu \mathrm{m}$ between the tines while each of the rings had inner and outer diameters of $190 \mu \mathrm{m}$ and $220 \mu \mathrm{m}$ respectively with a thickness of $10 \mu \mathrm{m}$. The resonators in both device configurations were driven and sensed capacitively. Actuation was achieved in the DETF configuration using parallel plates of equal dimensions $(260 \mu \mathrm{m}$ long, $6 \mu \mathrm{m}$ wide, $25 \mu \mathrm{m}$ thick) attached to either side of each fork as shown in fig. 3 while plates of nominal dimensions $120 \mu \mathrm{m}$ long, $6 \mu \mathrm{m}$ wide, $10 \mu \mathrm{m}$ thick were attached to the rings as shown in fig. 2. All experiments were carried out in a temperature and pressure controlled vacuum probe station. A schematic of the measurement setup is shown in figure 4 .

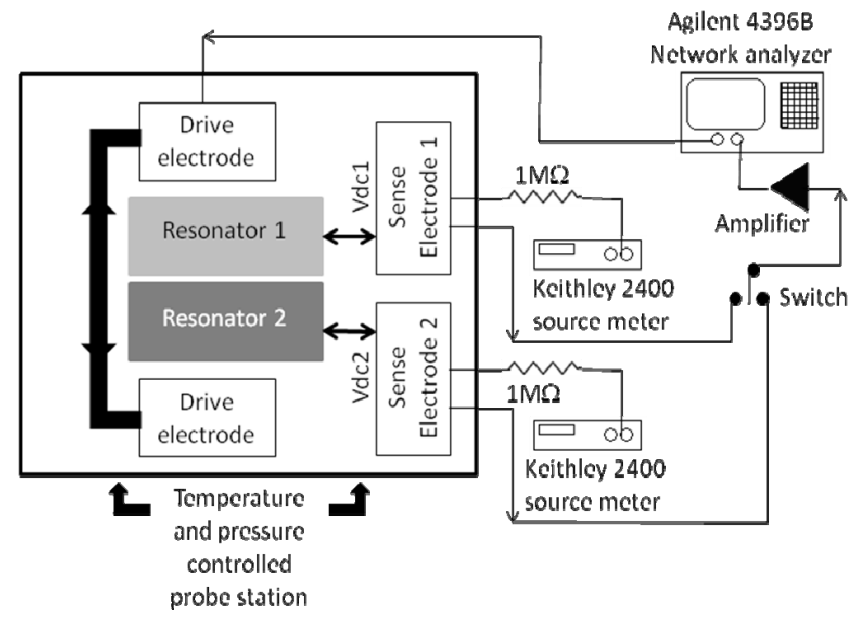

Figure 4: Schematic of measurement setup used for the characterization of the electrically coulped resonator pairs.

\section{B. Sensitivity analysis}

Initially, DC polarization voltages corresponding to +20 and $-20 \mathrm{~V}$ were initially applied on resonators 1 and 2 respectively of the ring configuration while maintaining the pressure and temperature constant at nearly $20 \mu$ Torr and $295 \mathrm{~K}$ $(+/-0.5 \mathrm{~K})$ respectively. This induces a negative electrostatic spring between the resonators without disrupting the initial mechanical symmetry. Maintaining the temperature and pressure of the ambient constant, the voltage on resonator 2 was then increased from $-20 \mathrm{~V}$ to $-5 \mathrm{~V}$ in steps of $5 \mathrm{~V}$ thereby altering the stiffness of resonator 2 relative to that of resonator 1 owing to the electrical spring softening effect. (It is to be noted that variations in the voltage of resonator 2 also alter the voltage difference between the resonators thereby varying the effective coupling spring constant along with the stiffness of resonator 2.) The variations in the eigenstates and resonant frequency owing to this induced perturbation in stiffness were then deduced from the $\mathrm{Y}$ parameter frequency responses of each of the two coupled resonators extracted from the S21 transmission frequency response measured on a Network Analyzer (refer fig. 4). The DC bias voltage normalized transmission responses of resonators 1 and 2 of the flexural wine glass mode ring configuration (after cancelling the effects of capacitive feed-through current) for the different bias conditions are shown in figs. 5(a) and 5(b) respectively.
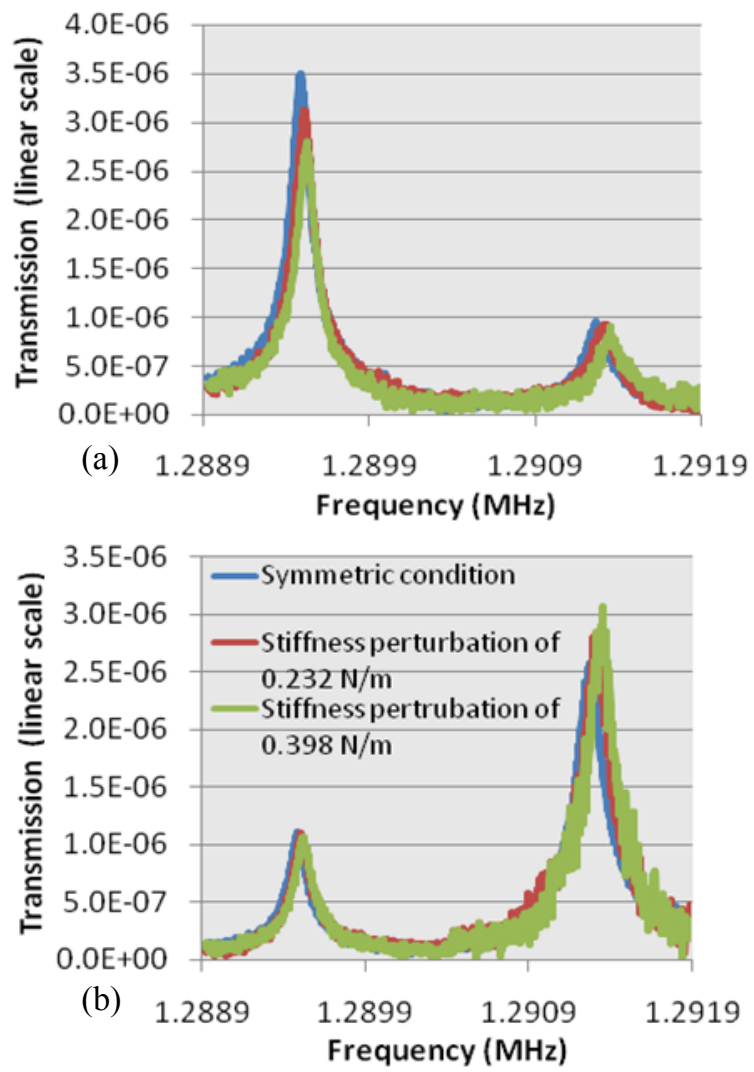

Figure 5: Experimentally observed (DC voltage normalized) transmission frequency response of resonator 1 (a) and resonator 2 (b).

Comparing the relative shifts in the eigenstates and those in resonant frequency (refer figs. 6 and 7), it can be noticed that for the same parametric stiffness variation, the relative shifts in the eigenstates of the system are over three orders of magnitude greater than those in resonant frequency. The effects of electrical spring tuning (due to variation in the difference in voltage between the two resonators) on the parametric sensitivity can also be observed from figure 6 . The variations in voltage allow for tuning the coupling spring 
constant and in effect, the parametric sensitivity of these devices (refer equation 1).

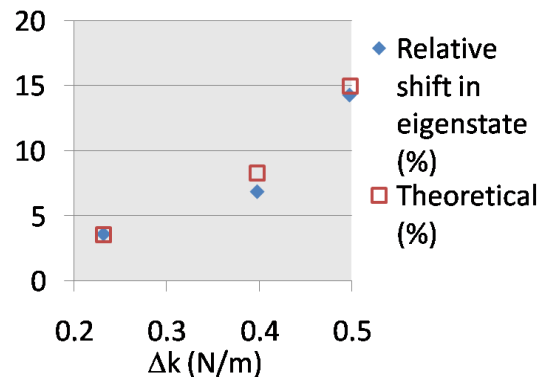

Figure 6: Relative shifts in the eigenstates for induced stiffness perturbations in resonator 2 .

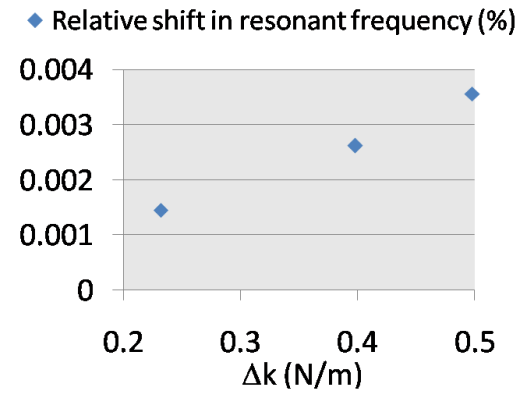

Figure 7: Experimentally observed relative shifts in the resonant frequency for the same induced perturbations in stiffness on resonator 2.

\section{Temperature test}

In order to study the common mode rejection capabilities, one of the coupled resonators is subjected to an electrostatically induced stiffness perturbation under varying ambient pressure and temperature and the variations in the eigenstates and resonant frequency are studied.

First, a temperature study was carried out by observing the variations in the eigenstates and resonant frequency for an induced stiffness perturbation of $.232 \mathrm{~N} / \mathrm{m}$ on resonator 2 of the flexural wine-glass mode ring configuration at varying ambient temperature. The sample stage temperature of the probe station was increased from $300 \mathrm{~K}(+/-0.5 \mathrm{~K})$ up to $340 \mathrm{~K}$ $(+/-0.5 \mathrm{~K})$ in steps of $20 \mathrm{~K}$. The pressure was maintained constant at $20 \mu$ Torr. The voltage normalized transmission responses of resonators 1 and 2 (after cancelling the effects of capacitive feed-through current) at the temperatures of $300 \mathrm{~K}$ and $320 \mathrm{~K}$, before and after inducing the stiffness perturbation are shown in figs. 8 and 9 respectively.

Figs. 10 and 11 compare the relative shifts in the eigenstates and those in resonant frequency for the same induced stiffness perturbation in resonator 2 under different ambient temperatures. It can be observed that while the shift in the resonant frequency (first eigenvalue) decreases with increasing temperature (for the same induced stiffness variation), the shifts in the measured eigenstate yield a relative insensitivity of nearly one order of magnitude.
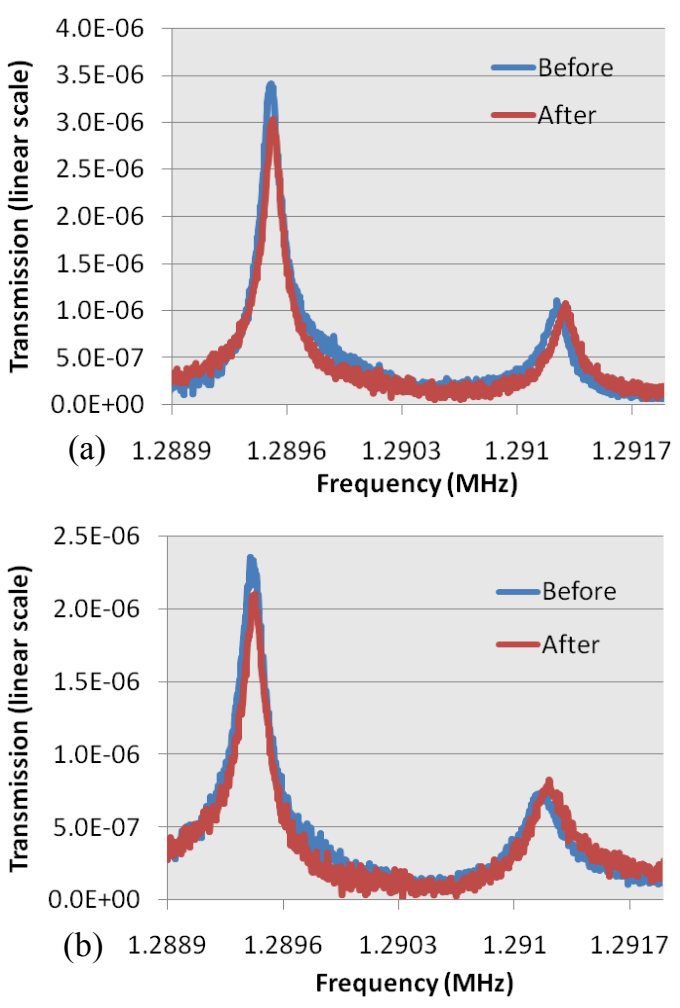

Figure 8: DC bias voltage normalized transmission response observed from resonator 1 before and after inducing a stiffness perturbation in resonator 2 at $300 \mathrm{~K}$ (a) and $320 \mathrm{~K}$ (b).
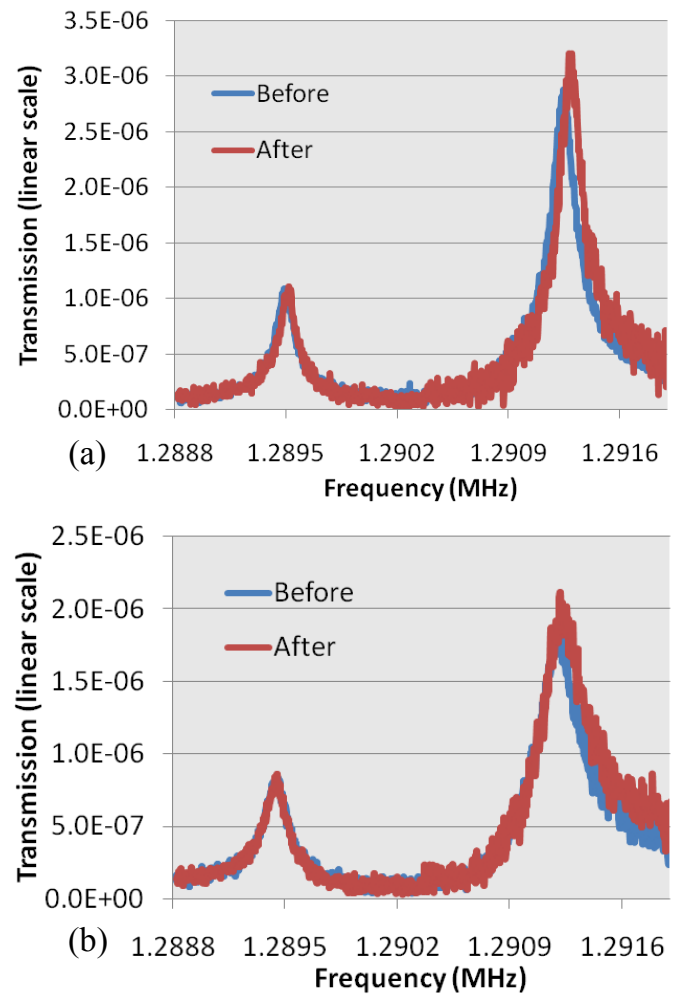

Figure 9: DC bias voltage normalized transmission response observed from resonator 2 before and after inducing a stiffness perturbation in resonator 2 at $300 \mathrm{~K}$ (a) and $320 \mathrm{~K}(\mathrm{~b})$. 


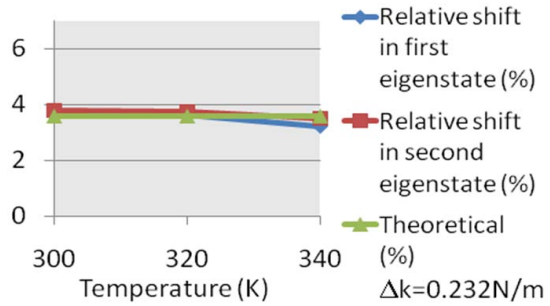

Figure 10: Experimentally observed relative shifts in the eigenstates for a stiffness perturbation of $0.232 \mathrm{~N} / \mathrm{m}$ at varying ambient temperature

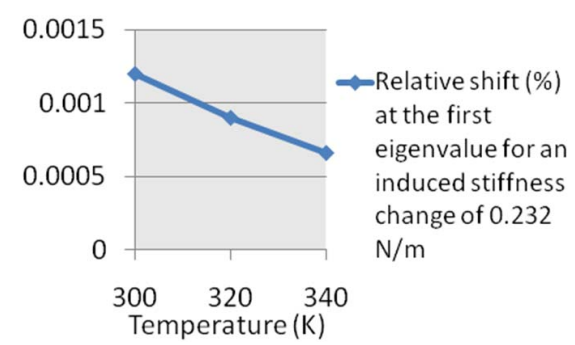

Figure 11: Experimentally observed relative shifts in the resonant frequency for the same stiffness perturbation of at varying ambient temperature.

\section{Pressure test}

Similar tests were carried out by keeping the temperature constant at $295 \mathrm{~K}(+/-0.5 \mathrm{~K})$ but varying the ambient pressure from $10 \mathrm{mTorr}$ to 1 Torr in order to determine the common mode rejection capability with variations in pressure. The DETF configuration (fig. 3) was chosen to perform the pressure tests in order to show the commonality of the concept to all coupled resonator topologies.

DC polarization voltages corresponding to $+5 \mathrm{~V}$ and $-5 \mathrm{~V}$ were initially applied on resonators 1 and 2 respectively of the DETF configuration. This should create the unperturbed symmetric condition of the initial eigenvalue problem. The voltage on resonator 2 was then increased to $+6 \mathrm{~V}$ and the corresponding variations in the eigenstates and resonant frequency were studied at varying ambient pressure for this induced stiffness perturbation in resonator 2 . The extracted transmission frequency responses from resonators 1 and 2 before and after inducing the stiffness perturbation on resonator 2 at three different pressure conditions of $10 \mathrm{mTorr}$, $100 \mathrm{mTorr}$ and 1 Torr are given in figs. 12 and 13 respectively. The relative shifts in the eigenstates and resonant frequency for the same induced stiffness perturbation at varying ambient pressure are shown in figs 14 and 15 respectively. It can be observed that while the relative shifts in the resonant frequency remain relatively constant up to about $100 \mathrm{mTorr}$, they change at higher ambient pressure. However, the relative shifts in the eigenstates remain relatively unaffected by fluctuations in the ambient pressure. This increased robustness to pressure fluctuations also suggests that measuring the shifts in the eigenstates in such sensors yield outputs that are relatively independent of the Quality factor of resonance. This suggests that the sensor resolution is no longer limited to the $\mathrm{Q}$ factor thereby providing an important advantage for sensor design. However, since the eigenstates are deduced from the amplitude of vibration of each the resonators at the fundamental modes of vibration, the resolution in such sensors is dictated by the amplitude noise in the system.
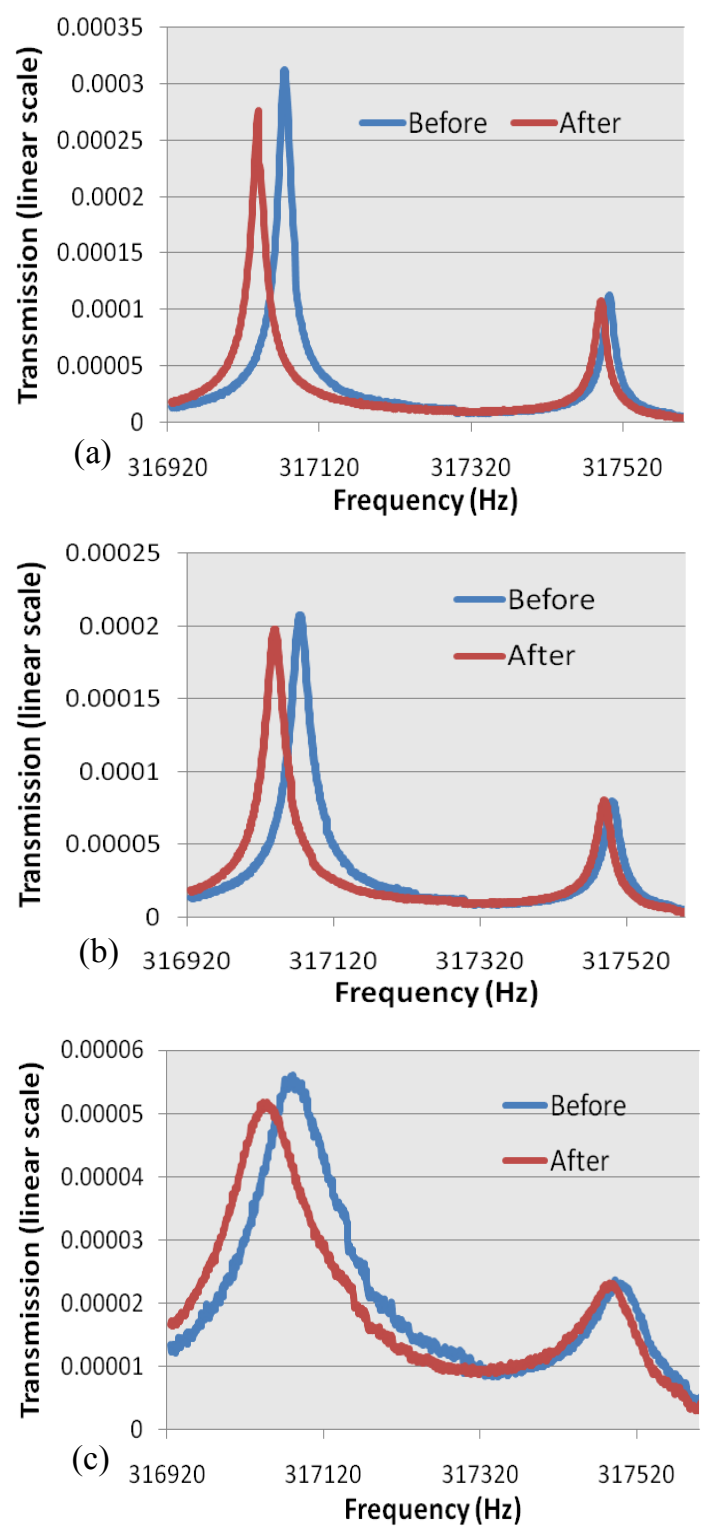

Figure 12: DC bias voltage normalized transmission response observed from resonator1 before and after inducing a stiffness perturbation in resonator 2 at $10 \mathrm{mTorr}(\mathrm{a}), 100 \mathrm{mTorr}$ (b) and 1 Torr (c).

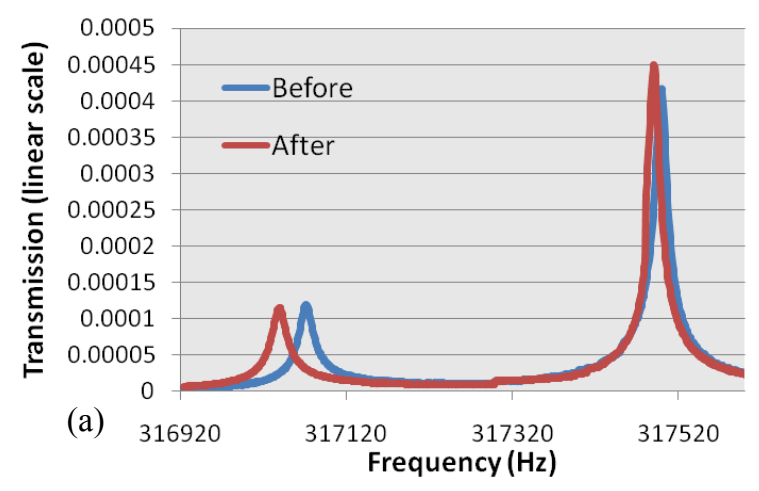



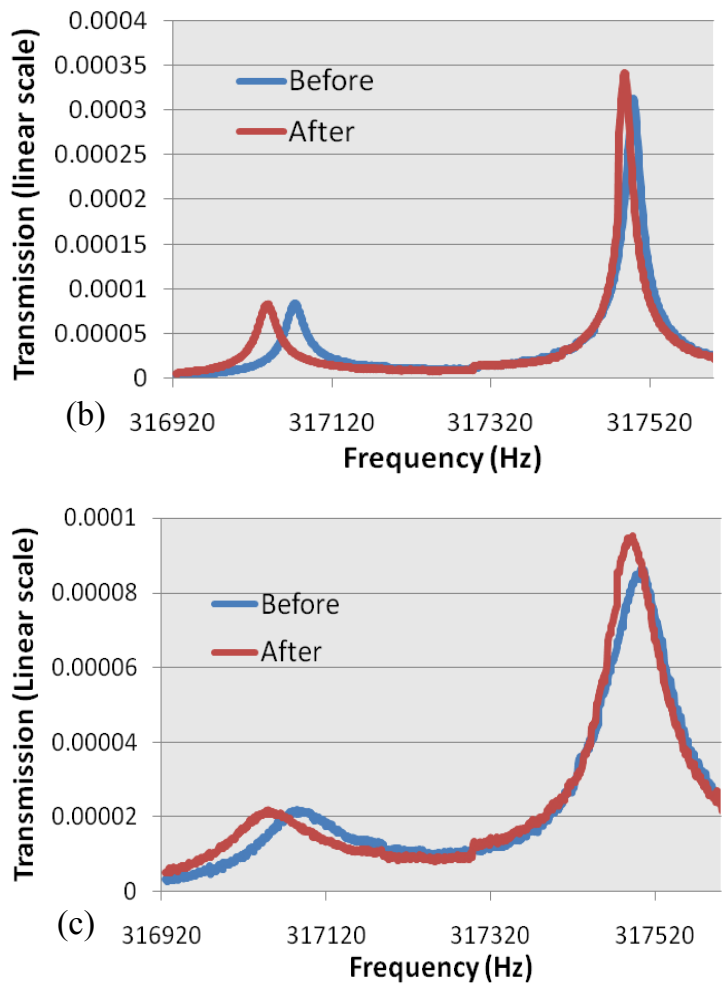

Figure 13: DC bias voltage normalized transmission response observed from resonator 2 before and after inducing a stiffness perturbation in resonator 2 at $10 \mathrm{mTorr}(\mathrm{a}), 100 \mathrm{mTorr}(\mathrm{b})$ and 1 Torr (c).

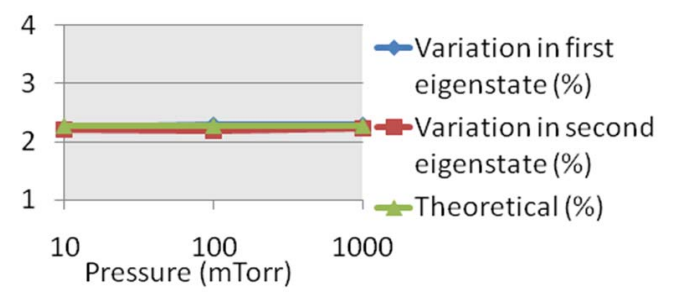

Figure 14: Experimentally observed relative shifts in the eigenstates for an induced stiffness perturbation on resonator 2 at varying ambient pressure

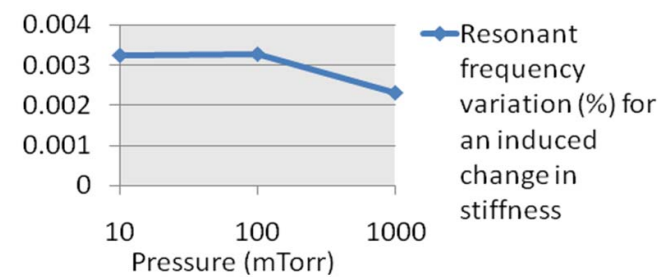

Figure 15: Experimentally observed relative shifts in the resonant frequency for the same stiffness perturbation at varying ambient pressure.

\section{CONCLUSION}

This paper demonstrates the common mode rejection capabilities of electrically coupled MEMS resonators utilizing mode localization for sensor applications. Relative shifts in the eigenstates that are over three orders of magnitude greater than corresponding resonant frequency variations for an induced perturbation in stiffness are experimentally demonstrated. The shifts are also shown to remain relatively unaffected by ambient drifts in temperature and pressure. A relative insensitivity in the output of nearly one order of magnitude compared to resonant frequency variations is experimentally demonstrated. Furthermore, the relative shifts in the eigenstates are shown to remain stable to variations in the pressure and the $\mathrm{Q}$ factor of resonance, making such an approach particularly attractive. This increased robustness and enhanced parametric sensitivity makes this approach highly sensitive to the measurand while being relatively insensitive to environmental drift.

\section{ACKNOWLEDGMENT}

The authors would like to thank Prof. Jim Woodhouse of the Cambridge University Engineering department (CUED) for useful discussions. The financial support offered by the British Council (UKIERI), Churchill college and Cambridge Commonwealth Trust is also gratefully acknowledged.

\section{REFERENCES}

[1] J. E.-Y. Lee, B. Bahreyni, Y. Zhu, and A. A. Seshia, "Ultrasensitive mass balanced based on a bulk acoustic mode single-crystal silicon resonator," Appl. Phys. Lett., vol. 91, pp. 234103, 2007.

[2] E.P. Quevy, and R.T. Howe, "MEMS resonators for precise reference oscillators," in Radio Frequency Integrated Circuits Symposium, pp. 113-116, 2005.

[3] W.-T. Hsu, J.R. Clark, and C.T.-C. Nguyen, "Mechanically temperature-compensated flexural-mode micromechanical resonators", IEEE International Electron Devices Meeting, 399-402, 2000.

[4] R. G. Azevedo, W. Huang, O. M. O'Reilly, and A. P. Pisano, "Dualmode temperature compensation for a comb-driven MEMS resonant strain gauge," Sens. Actuators, Phys. A, vol. 144, pp. 374-380, 2008.

[5] M.A. Hopcroft, M. Agarwal, K.K. Park, B. Kim, C.M. Jha, R.N. Candler, G. Yama, B. Murmann, and T.W. Kenny, "Temperature Compensation of a MEMS Resonator Using Quality Factor as a Thermometer," in Proc. $19^{\text {th }}$ IEEE Int. Conf. Microelectromech. Syst., pp. 222-225, 2006.

[6] M. Spletzer, A. Raman, H. Sumali, and J. P. Sullivan, "Highly sensitive mass detection and identification using vibration localization in coupled microcantilever arrays,” Appl. Phys. Lett., vol. 92, pp. 114102, 2008.

[7] M. Spletzer, A. Raman, Al. Q. Wu, X. Xu, and R. Reifenberger, "Ultrasensitive mass sensing using mode localization in coupled microcantilevers," Appl. Phys. Lett., vol. 88, pp. 254102, 2006.

[8] P. Thiruvenkatanathan, J. Yan, J.E-Y. Lee, A.A. Seshia, "Enhancing parametric sensitivity using mode localization in electrically coupled MEMS resonators", $15^{\text {th }}$ Int. Conf. on Solid-State Sens., Actuators and Microsyst., Transducers, 2009, in press.

[9] S. Pourkamali, and F. Ayazi, "Electrically coupled MEMS bandpass filters Part II. Without coupling element," Sens. Actuators, Phys. A, vol. 122 , pp. 317-325, 2005. 